\title{
ANALYSIS OF NONLOCAL ELECTROSTATIC EFFECTS IN CHIRAL SMECTIC C LIQUID CRYSTALS
}

\author{
By
}

Jinhae Park

and

\section{Carme Calderer}

\section{IMA Preprint Series \# 2109}

(March 2006)

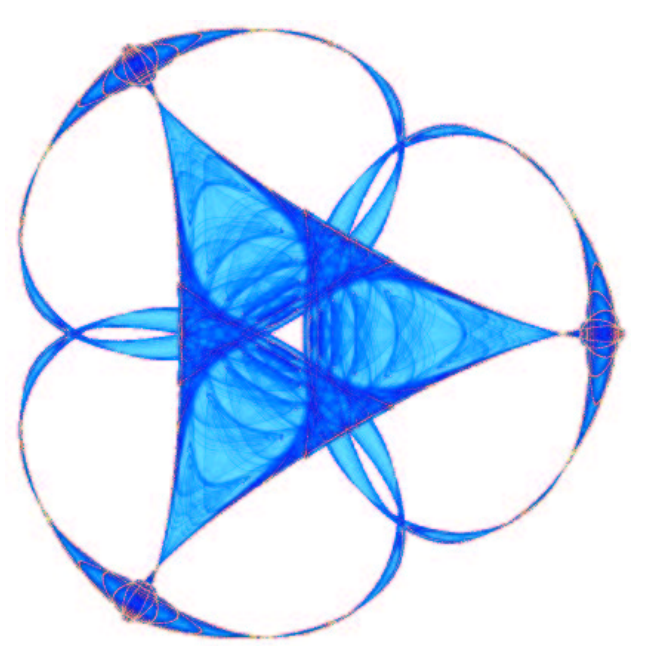

INSTITUTE FOR MATHEMATICS AND ITS APPLICATIONS

UNIVERSITY OF MINNESOTA

400 Lind Hall

207 Church Street S.E.

Minneapolis, Minnesota 55455-0436

Phone: 612/624-6066 Fax: 612/626-7370

URL: http://www.ima.umn.edu 


\title{
ANALYSIS OF NONLOCAL ELECTROSTATIC EFFECTS IN CHIRAL SMECTIC C LIQUID CRYSTALS*
}

\author{
JINHAE PARK ${ }^{\dagger}$ AND M. CARME CALDERER ${ }^{\dagger}$
}

\begin{abstract}
We present modeling and analysis of smectic C phases of liquid crystals capable of sustaining spontaneous polarization. The layered liquid crystals are also assumed to be chiral. We study minimization of the total energy subject to electrostatic constraints. In order to determine mathematically and physically relevant boundary conditions, we appeal to the analogy between the current problem and the vorticity in fluids. We place a special emphasis on the nonlocal and selfenergy effects arising from spontaneous polarization. We discuss examples pertaining to the electric field created by the liquid crystal in dielectric media, and also to the possible role of a domain shape as an energy reduction mechanism.
\end{abstract}

Key words. Chiral, energy minimizer, electrostatic self-interaction, ferroelectricity, helical filaments, liquid crystals, nonlocal effects, smectic C phase, smectic layers, vortex tubes.

AMS subject classifications. 35Q35, 47J30, 49J40, 49J45, 74A35, 76A15, 82B21, 82B26, 82D45

1. Introduction. This article analyzes nonlocal electrostatic effects associated with polarized states of liquid crystals. We assume that the liquid crystals are of smectic type, possess spontaneous polarization, and may also be chiral. We study minimization of the total energy in $\mathbf{R}^{3}$, subject to electrostatic constraints.

In smectic liquid crystals, centers of mass of molecules are arranged locally in one-dimensional layers described by a complex field $\psi=\rho e^{i \omega}$; level sets of the phase function $\omega$ denote layer locations, with $\nabla \omega$ being parallel to the layer normal. Nonparallel unit vector fields $\mathbf{n}$ and $\mathbf{p}$ describe the orientational ordering of biaxial molecules. Another feature of smectic $\mathrm{C}$ phases is that the director $\mathbf{n}$ makes a preferred angle $\alpha$ with the layer normal vector. The angle $\alpha$ is a temperature and material dependent quantity ranging typically from 0 to $\frac{\pi}{4}$. We visualize smectic $\mathrm{C}$ phases in terms of cones with axis along the layer normal and semi-angle $\alpha$. The director $\mathbf{n}$ is then parallel to a generating straight line of the cone. Since the systems that we consider are ferroelectric, that is, they have spontaneous polarization, we take $\mathbf{p}$ to be parallel to the polarization field $\mathbf{P} ; \mathbf{n}$ corresponds to the uniaxial director measuring the average alignment of molecular long axes of either rod-like or bent-core molecules [33, 34]. The electrostatic potential $\varphi$ is also a variable of the problem.

Many different types of liquid crystals form smectic C phases (i.e., one-dimensional layer structures). The earlier low molecular weight liquid crystals labelled as smectic C [25] owe ferroelectricity to the molecular dipoles associated with side chains. In such cases, the direction of $\mathbf{P}$ is determined and tends to be perpendicular to $\mathbf{n}$ and $\nabla \omega$ (This type of ferroelectricity, known as improper [21], is absent in the smectic A phase due to $\mathbf{n}$ being parallel to $\nabla \omega)$. Since the magnitude of the polarization is usually small, studies of such liquid crystals normally neglect nonlocal electric field effects, and assign constant values to applied fields. Many of the low molecular weight smectic C liquid crystals are cholesteric. On the other hand, B2 phases of bent-core molecule liquid crystals are mostly non-chiral and have large polarization values. Although $\mathbf{P}$ tends to be perpendicular to $\mathbf{n}$, the plane determined by these vectors is

\footnotetext{
${ }^{\dagger}$ School of Mathematics, University of Minnesota, Minneapolis, MN 55455, USA (Email: jinhae@math.umn.edu and mcc@math.umn.edu, Fax:+1-612-626-2017).

*This work is partially supported by the National Science Foundation, grant number DMS0128832 .
} 
free to rotate about $\mathbf{n}$. Unlike in the case of small molecule smectics, the B2 phases are not subject to the constraint of $\mathbf{P}$, that is parallel to $\nabla \omega \times \mathbf{n}$. However, analysis of the latter requires accounting for nonlocal energy.

Throughout this work, we will assume that the liquid crystals are chiral and use the conventional notation $\mathrm{C}^{*}$ to denote such chiral smectic phases. An important bulk configuration of chiral smectic $C$ phases is that the variables $\mathbf{n}$ and $\mathbf{p}$ rotate spatially around the axis of the previously described cone, with $\mathbf{n}$ being parallel to a generating straight line of the cone. This accordingly results in zero net polarization over a helical pitch. In suppressing the helix by applying an external electric field or boundary conditions, ferroelectric states with opposite polarization emerge. The transition between the chiral state and the ferroelectric ones is not regarded as a typical phase transition; however, it is at the core of device applications of ferroelectric liquid crystals. Mathematically, we address in previous work [27] some stability properties with respect to boundary conditions and electric fields, in the case that nonlocal effects are neglected.

The total energy we analyze consists of nematic, smectic, Ginzburg-Landau, electrostatic, and surface contributions. The nematic and smectic free energy densities follow the forms of Oseen-Frank, de Gennes, and Chen-Lubensky that penalize departure from preferred molecular alignment and orientation with respect to the layers. Other macroscopic theories of smectic $\mathrm{C}$ phases can be found in the literature $[23,33,37]$. Our choice is motivated by the covariant structure of the Lubensky form; this is quadratic in second order gradients of $\psi$ and especially amenable to treatment by calculus of variations. We relax the constraint $\mathbf{p} \perp\{\mathbf{n}, \nabla \omega\}$, and instead incorporate a penalty energy into the model. The Ginzburg-Landau energy tends to select a preferred magnitude of the polarization according to temperature and material parameters. The current energy is appropriate to model B2 ferroelectric phases provided that we omit the previously mentioned penalty term and chirality.

The electrostatic energy comprises a dielectric contribution and a ferroelectric one. The latter accounts for the energy of self-interaction between the polarization and its own electric field, as well as the electrostatic energy outside the liquid crystal domain. So far, this situation is analogous to that of a ferroelectric solid. However, there are some fundamental differences between these two behaviors. In the solid, the directions of polarizations are determined by lattice directions; the latter are difficult to alter by an external field. The "softness" of a liquid crystal allows for changes in molecular alignments so as to reduce the energy. For instance, in the case of a liquid crystal located between conducting plates, the distribution of polarization is such that it gets compensated by free charges in the conductor, inducing a zero electric field outside. As a result, the nonlocal energy vanishes. Suppressing the helix in the smectic $\mathrm{C}^{*}$ phase is a mechanism of reducing nonlocal energy. In a related work [19], Khachaturyan showed that a polarized homogeneous state of the nematic phase is unstable with respect to a slight dipole-dipole interaction, resulting in a symmetry breaking.

If a B2 liquid crystal is embedded in another liquid, such as its own isotropic phase, it may actually change its shape so that the nonlocal energy is zero. This gives a good explanation for the telephone-cord shape observed in many ferroelectric liquid crystal filaments $[5,8,16,17]$. Modeling of static liquid crystal helical filaments can be found in $[2,36]$. Chevron structures with alternating domains of opposite polarization are also found in some materials [5]. The phenomena of changing shape to reduce electrostatic energy has also been observed in thin polarized piezoelectric films (for 
example, $\mathrm{ZnO}$ nanobelt [20]).

In the current analysis, defects are not included. In particular, we assume that $\nabla \omega$ is defined everywhere on $\partial \Omega$ with possible exceptions on the edges of $\Omega$. This, in turn, determines the type of the domain occupied by the liquid crystal, and the nature of boundary conditions on the phase function $\omega$. We make use of analogies with the geometry of vortex tubes and sheets in fluid mechanics. Indeed, we take $\Omega$ to be a cylinder-like domain analogous to a vortex tube which has the lateral surface $\Sigma$ corresponding to a vortex sheet, and is contained between surfaces $\mathcal{S}_{1}$ and $\mathcal{S}_{2}$. We deal with two types of boundary conditions on $\omega$ : one corresponding to the layer structure reaching the boundary in a tangential fashion, and the other with layers being perpendicular to the boundary. The latter correspond to the geometry of the Clark-Lagerwall effect in ferroelectric displays [4, 14, 39]. We also prescribe the electrostatic potential on a part of the boundary. These boundary conditions together with assumptions for the constitutive parameters (see section 3) allow us to prove existence of minimizers of the total energy by using direct methods of calculus of variations. One important issue is whether the minimizers thus obtained correspond to chiral structures or ferroelectric ones. We apply asymptotic analysis to obtain a classification of minimizers [1].

In [18], Joo and Phillips studied the phase transitions between chiral nematic, smectic $\mathrm{A}^{*}$, and $\mathrm{C}^{*}$ liquid crystals, and carried out extensive stability analyses. Their work gives a rigorous classification of the energy minimizing phase regimes. Another important merit of the article is establishing the coercivity of the smectic $\mathrm{C}^{*}$ energy for the first time. For a mathematical analysis of the phase transitions between the chiral and smectic $A^{*}$ liquid crystals with focus on the analogies of the phase transition between conductor and superconductor, the reader is referred to [1]. Studies of periodic ferroelectric and antiferroelectric phases, and analysis of time dependent problems arising in switching are also carried out by the authors [28]. Experimental treatments and studies of smectic $\mathrm{C}^{*}$ liquid crystals including the influence of an electric field are found in $[10,11,12,29,30,31]$. For structural understanding and modeling of ferroelectricity, we refer to the books by Goody et al [13], Lagerwall [21], Pikin [32], and by Muševič, Blinc and Žekš [26].

This article is organized as follows: In section 2, we present free energy functions of smectic $\mathrm{C}^{*}$ materials with concentration on the polarization and electrostatic energies. We discuss constraint relaxation and approximations of the electrostatic energies. In section 3, we prove existence of minimizers and study examples regarding the relationship between domain shapes and polarizations. We also present two different versions of the variational problem, with one of them corresponding to a liquid crystal placed between metalic plates. In the other formulation, the liquid crystal is placed in a dielectric media, subject to the electric field generated by the material polarization. In section 4, we carry out asymptotic studies of the minimizers obtained in section 3 to determine whether they correspond to chiral or ferroelectric structures. We outline some conclusions in section 5 .

2. Free energy functions. We present the energy functional for smectic materials to be analyzed. This includes nematic, smectic, Ginzburg-Landau, surface, and electric contributions. We will also show how they give rise to simple forms found in the literature.

Equilibrium configurations of smectic $\mathrm{C}^{*}$ liquid crystals occupying a smooth domain $\Omega$ in $\mathbf{R}^{3}$ are given by quadruples $(\psi, \mathbf{n}, \mathbf{P}, \mathbf{E})$ of fields, $\psi: \Omega \rightarrow \mathbb{C}, \mathbf{n}: \Omega \rightarrow \mathbf{S}^{2}$, 
$\mathbf{P}: \Omega \rightarrow \mathbf{R}^{3}$, and $\mathbf{E}: \mathbf{R}^{3} \rightarrow \mathbf{R}^{3}$, that are critical points of the total energy functional

$$
\begin{aligned}
\mathcal{E}(\mathbf{n}, \mathbf{P}, \psi, \mathbf{E})= & \int_{\Omega}\left\{F_{N}(\mathbf{n}, \mathbf{P}, \nabla \mathbf{n})+F_{S m}\left(\nabla \mathbf{n}, \nabla \psi, \nabla^{2} \psi\right)\right. \\
& \left.+F_{P}(\mathbf{n}, \mathbf{P}, \nabla \mathbf{P}, \nabla \psi)\right\} d \mathbf{x}+\int_{\partial \Omega} F_{S}(\mathbf{n}, \nu) d S \\
+ & \int_{\mathbf{R}^{3}} F_{E}\left(\mathbf{n} \chi_{\Omega}, \mathbf{P} \chi_{\Omega}, \mathbf{E}\right) d \mathbf{x},
\end{aligned}
$$

subject to Maxwell's equations

$$
-\nabla \cdot \mathbf{D}=0, \quad \nabla \times \mathbf{E}=\mathbf{0} \text { in } \Omega,
$$

where $\mathbf{D}$ is the electric displacement vector, $\mathbf{E}$ is the electric field, and the functions $F_{N}, F_{S m}, F_{E}, F_{S}, F_{P}$ represent the Oseen-Frank, the smectic, the electrostatic, the surface anchoring ([6], p. 99), and the Ginzburg-Landau energy densities, respectively. $\chi_{\Omega}$ denotes the characteristic function.

2.1. Dielectric, nonlocal, and self-interaction terms. The electrostatic energy density in $\mathbf{R}^{3}[7,22]$ is given by

$$
\begin{aligned}
& F_{E}=-\frac{1}{2}\left((\varepsilon \mathbf{E} \cdot \mathbf{E}) \chi_{\Omega}+(\mathbf{E} \cdot \mathbf{E}) \chi_{\Omega^{c}}\right)-(\mathbf{P} \cdot \mathbf{E}) \chi_{\boldsymbol{\Omega}}, \\
& { }^{3} \mathbf{D}=\varepsilon \mathbf{E} \chi_{\boldsymbol{\Omega}}+\mathbf{E} \chi_{\boldsymbol{\Omega}^{\mathbf{c}}}+\mathbf{P} \chi_{\boldsymbol{\Omega}}, \\
& \varepsilon=\varepsilon_{\perp} \mathbf{I}+\varepsilon_{a} \mathbf{n} \otimes \mathbf{n},
\end{aligned}
$$

where $\varepsilon, \varepsilon_{\perp}$, and $\varepsilon_{a}$ represent the susceptibility tensor, dielectric permittivity, and dielectric anisotropy, respectively.

Note that (2.3) can be written as,

$$
F_{E}=-\frac{1}{2}\left[\left(\varepsilon_{\perp}|\mathbf{E}|^{2}+\varepsilon_{a}(\mathbf{n} \cdot \mathbf{E})^{2}+|\mathbf{E}|^{2} \chi_{\Omega^{c}}\right]-(\mathbf{P} \cdot \mathbf{E}) \chi_{\Omega} .\right.
$$

So, the electrostatic energy is given by

$$
\begin{aligned}
\int_{\mathbf{R}^{3}} F_{E} d \mathbf{x}= & -\frac{1}{2} \int_{\Omega}\left\{\varepsilon_{\perp}|\mathbf{E}|^{2}+\varepsilon_{a}(\mathbf{E} \cdot \mathbf{n})^{2}\right\} d \mathbf{x} \\
& -\frac{1}{2} \int_{\mathbf{R}^{3}-\Omega}|\mathbf{E}|^{2} d \mathbf{x}-\int_{\Omega} \mathbf{P} \cdot \mathbf{E} d \mathbf{x} .
\end{aligned}
$$

The two terms in the last row in (2.7) correspond to the self-interaction and nonlocal electrostatic energies, respectively. The term in the first row gives the dielectric contribution.

2.2. Nematic and Smectic free energies. The Oseen-Frank free energy density is given by

$$
\begin{aligned}
F_{N}= & K_{1}(\nabla \cdot \mathbf{n})^{2}+K_{2}(\mathbf{n} \cdot \nabla \times \mathbf{n}+\tau)^{2}+K_{3}|\mathbf{n} \times(\nabla \times \mathbf{n})+\gamma \mathbf{P}|^{2} \\
& +\left(K_{2}+K_{4}\right)\left(\operatorname{tr}(\nabla \mathbf{n})^{2}-(\nabla \cdot \mathbf{n})^{2}\right) .
\end{aligned}
$$

\footnotetext{
${ }^{3}$ We note that the electric dispacement $\mathbf{D}$ is usually written by $\mathbf{D}=\varepsilon \mathbf{E} \chi_{\Omega}+\mathbf{E} \chi_{\Omega^{c}}+4 \pi \mathbf{P}$. For simplicity, we represent $4 \pi \mathbf{P}$ by $\mathbf{P}$ in this paper.
} 
where $K_{i}, i=1,2,3,4$, denote elasticity constants. The scalar $\tau$ represents the chiral pitch of the helical structure of the cholesteric phases [9], and $K_{3} \gamma^{2}|\mathbf{P}|^{2}$ is an intrinsic bending stress ([7], p. 384). Here, $\gamma$ is a parameter included for the purpose of dimensionalization; hereafter, we will take $\gamma$ to be 1 . Such a term appears only in connection with the modeling of the smectic $\mathrm{C}^{*}$ since nematics with intrinsic bending have not been observed. Both quantities result from the loss of mirror symmetry of the smectic $\mathrm{C}^{*}$ phases. The fourth term in $F_{\mathrm{N}}$ is a null-Lagrangian; its integral is determined by $\mathbf{n}$ on $\partial \Omega$. The classical Oseen-Frank energy corresponds to the case that $\mathbf{P}$ is zero. Existence and regularity of minimizers for the classical Oseen-Frank energy were studied by R. Hardt et al [15].

The free energy density associated with the smectic layering follows the covariant form presented in [3]:

$$
\begin{aligned}
F_{S m}= & D\left(\mathbb{D}^{2} \psi\right)\left(\mathbb{D}^{2} \psi\right)^{*}+\left[C_{||} n_{i} n_{j}+C_{\perp}\left(\delta_{i j}-n_{i} n_{j}\right)\right]\left(\mathbb{D}_{i} \psi\right)\left(\mathbb{D}_{j} \psi\right)^{*} \\
& +r|\psi|^{2}+\frac{g}{2}|\psi|^{4} .
\end{aligned}
$$

with $\mathbb{D} \equiv \nabla-i q \mathbf{n}, q$ is the modulation wave number of the smectic layer, and $r=$ $a\left(T-T^{*}\right), a>0$; here $T$ denotes the (constant) temperature of the material and $T^{*}$ is the transition temperature from nematic to smectic. Model (2.9) yields the de Gennes model for Smectic $\mathrm{A}^{*}$ when $C_{\|}-C_{\perp}=0$ and $D=0$. The smectic $\mathrm{C}$ phase is characterized by $C_{\perp}<0$. Moreover, $C_{\perp} \geq 0$ in the smectic $\mathrm{A}^{*}$ and $C_{\perp}=0$ characterizes the transition to smectic C. Equivalently, the energy (2.9) can also be written as follow:

$$
F_{S m}=D\left|\mathbb{D}^{2} \psi\right|^{2}+C_{\perp}|\mathbb{D} \psi|^{2}+C_{a}|\mathbf{n} \cdot \mathbb{D} \psi|^{2}+r|\psi|^{2}+\frac{g}{2}|\psi|^{4} .
$$

Remark. The first term in (2.9) is obtained from [24] and it is a modification of $D_{\perp}\left(\delta_{i j}-n_{i} n_{j}\right)\left(\delta_{k l}-n_{k} n_{l}\right)\left(\mathbb{D}_{i} \mathbb{D}_{j}\right)\left(\mathbb{D}_{k} \mathbb{D}_{l}\right)^{*}$ in the original Chen-Lubensky model. The purpose of introducing the new term is to obtain coercivity of the energy. This fact was first observed in [18].

2.3. Anchoring energy. The anchoring energy is the Rapini-Papoular surface energy $[7,21]$ given by

$$
F_{S}=\omega_{n}\left(1-\alpha_{0}(\mathbf{n} \cdot \nu)^{2}\right),
$$

where $\omega_{n}$ and $\left|\alpha_{0}\right|<1$ are material constants, and $\nu$ denotes the unit normal to the surface. Note that the surface energy due to the polarization is not explicitly included in (2.11). In fact, the role of such a surface energy is an approximation to the nonlocal energy in (2.7), which we explicitly include in the problem.

2.4. Ginzburg-Landau energy with relaxation. The energy associated with the phase transition to the ferroelectric phases is given by the Ginzburg-Landau expression [22], $a_{0}|\mathbf{P}|^{2}+b_{0}|\mathbf{P}|^{4}$, where $b_{0}>0$ and $a_{0}=\alpha\left(T-T_{c}\right)$. Ferroelectric phases correspond to the case $T<T_{c}$.

In contrast to solids, the direction of polarization in many liquid crystals is determined by the direction $\mathbf{n}$ and the layer normal $\nabla \omega$. In fact, a symmetry argument shows that $\mathbf{P}$ is perpendicular to both $\mathbf{n}$ and $\nabla \omega$. For this reason, we express $\mathbf{P}$ as follows ([7], p. 384):

$$
\mathbf{P}=\left\{\begin{array}{cl}
|\mathbf{P}| \frac{\nabla \omega \times \mathbf{n}}{|\nabla \omega \times \mathbf{n}|} & \text { if } \nabla \omega \times \mathbf{n} \neq \mathbf{0} \\
\mathbf{0} & \text { if } \nabla \omega \times \mathbf{n}=\mathbf{0}
\end{array}\right.
$$


Therefore, the Ginzburg-Landau energy with relaxation is given by

$$
F_{P}=B|\nabla \mathbf{P}|^{2}+a_{0}|\mathbf{P}|^{2}+b_{0}|\mathbf{P}|^{4}+\frac{1}{\epsilon^{2}}|(|\nabla \omega \times \mathbf{n}|) \mathbf{P}-| \mathbf{P}|(\nabla \omega \times \mathbf{n})|^{2},
$$

where $B>0, a_{0}<0, b_{0}>0$ and $\epsilon>0$. Here, we note that the last term in (2.13), $\frac{1}{\epsilon^{2}}|(|\nabla \omega \times \mathbf{n}|) \mathbf{P}-| \mathbf{P}|(\nabla \omega \times \mathbf{n})|^{2}$, is a penalty term for $(2.12)$ and $|\nabla \mathbf{P}|^{2}$ is a regularizing term.

2.5. Electrostatic approximations. The presence of polarization in the sample causes a point charge density $\rho_{p}=-\nabla \cdot \mathbf{P}$ in the bulk, and $\sigma=\mathbf{P} \cdot \nu$ where $\sigma$ is the surface density of charges [38]. This will help us interpret the energies $\int_{\mathbf{R}^{3}-\Omega}|\mathbf{E}|^{2} d \mathbf{x}$ and $\int_{\Omega} \mathbf{P} \cdot \mathbf{E} d \mathbf{x}$. For this, let us consider the special case that $\Omega$ is a ball of radius $r_{0}$ centered at $\mathbf{0}$, with the constant surface charge density $\sigma$. We calculate the electric potential $\varphi[38]$ as

$$
\varphi(\mathbf{x})= \begin{cases}\frac{\sigma r_{0}^{2}}{|\mathbf{x}|} & \text { if }|\mathbf{x}|>r_{0} \\ \sigma r_{0} & \text { if }|\mathbf{x}| \leq r_{0}\end{cases}
$$

Then for $|\mathbf{x}|>r_{0}$, we get

$$
|\mathbf{E}(r)|=\frac{\sigma r_{0}^{2}}{r^{2}}
$$

and hence

$$
\begin{aligned}
\int_{\mathbb{R}^{3}-\Omega}|\mathbf{E}|^{2} d \mathbf{x} & =\int_{0}^{2 \pi} \int_{0}^{\pi} \int_{r_{0}}^{\infty} \frac{\sigma^{2} r_{0}^{4}}{r^{2}} \sin \phi d r d \phi d \theta \\
& =r_{0} \int_{\partial \Omega}(\mathbf{P} \cdot \nu)^{2} d S .
\end{aligned}
$$

This explains why the term $\int_{\partial \Omega}(\mathbf{P} \cdot \nu)^{2} d S$ appears in liquid crystal models [21].

Now, let us consider the self-interaction energy. For our illustration, we consider the case that $\varepsilon_{a}<<\varepsilon_{\perp}$, and we neglect $\varepsilon_{a}$ in the model. It then follows that the self-interaction energy is related with the Coulomb energy by

$$
\int_{\Omega} \int_{\Omega} \frac{\nabla \cdot \mathbf{P}(\mathbf{x}) \nabla \cdot \mathbf{P}(\mathbf{y})}{|\mathbf{x}-\mathbf{y}|} d \mathbf{x} d \mathbf{y}=-4 \pi \varepsilon_{\perp} \int_{\Omega} \mathbf{P} \cdot \mathbf{E} d \mathbf{x} .
$$

We point out that the Coulomb energy is often approximated by the polar energy $\int_{\Omega}(\nabla \cdot \mathbf{P})^{2} d \mathbf{x}$ found in the literature $[7,21]$. We summarize the total energy involved in the electrostatic approximation without an external field as follows:

$$
\begin{aligned}
\mathcal{E}(\mathbf{n}, \mathbf{P}, \psi) & =\int_{\Omega}\left\{F_{N}+F_{S m}+G_{P}\right\} d \mathbf{x}+\int_{\partial \Omega} G_{S} d S, \\
G_{S}= & \omega_{p}\left(1-\frac{\mathbf{P} \cdot \nu}{|\mathbf{P}|}\right)+\omega_{r}\left(1-\frac{(\mathbf{P} \cdot \nu)^{2}}{|\mathbf{P}|^{2}}\right) \\
& +\omega_{n}\left(1-(\mathbf{n} \cdot \nu)^{2}\right), \\
G_{P} & =F_{P}+B_{1}(\nabla \cdot \mathbf{P})^{2} .
\end{aligned}
$$

Minimization of the energy (2.14) is studied in previous work [27]. 
3. Existence of minimizers. In this section, we study the boundary conditions for smectic-C* layer configurations, and prove existence of minimizers. We also discuss applications and provide examples that explain the relationship between domain shape and ferroelectricity.

Throughout this paper, we assume that the constitutive parameters satisfy

$$
\begin{aligned}
& g>0, \quad q \geq 0, \tau \geq 0, r<0, b_{0}>0, \omega_{n}>0, \\
& D>0, C_{\perp}<0, C_{a}>0, c_{1} \geq K_{2}+K_{4} \geq c_{0}, \\
& K_{1} \geq K_{2}+K_{4}, K_{3} \geq K_{2}+K_{4}, 0 \geq K_{4}
\end{aligned}
$$

where $c_{0}$ and $c_{1}$ are positive constants. The latter inequalities are necessary conditions to ensure coercivity of the energy [1].

3.1. Boundary conditions. Let $\Omega \subset \mathbf{R}^{3}$ be a bounded, cylinder-like domain, with boundary $\partial \Omega=\Sigma \cup \mathcal{S}_{1} \cup \mathcal{S}_{2}$. We assume that the lateral surface $\Sigma$ is of class $C^{2}$, and that $\mathcal{S}_{1}$ and $\mathcal{S}_{2}$ are plane cross sections with unit normal $\nu_{1}$ and $\nu_{2}$, respectively. Letting $\psi=\rho e^{i \omega}$, we rewrite $F_{S m}$ as

$$
\begin{aligned}
F_{S m}= & D\left|\mathbb{D}^{2} \psi\right|^{2}+C_{\perp}|\mathbb{D} \psi|^{2}+C_{a}|\mathbf{n} \cdot \mathbb{D} \psi|^{2}+r|\psi|^{2}+\frac{g}{2}|\psi|^{4} \\
= & D\left[\left(\Delta \rho-\rho|\nabla \omega-q \mathbf{n}|^{2}\right)^{2}+(\rho \nabla \cdot(\nabla \omega-q \mathbf{n})\right. \\
& \left.+2 \nabla \rho \cdot(\nabla \omega-q \mathbf{n}))^{2}\right]+C_{\perp}\left[|\nabla \rho|^{2}+\rho^{2}|\nabla \omega-q \mathbf{n}|^{2}\right] \\
& +C_{a}\left[(\nabla \rho \cdot \mathbf{n})^{2}+\rho^{2}(\nabla \omega \cdot \mathbf{n}-q)^{2}\right]+r \rho^{2}+\frac{g}{2} \rho^{4} .
\end{aligned}
$$

The following lemma based on Gauss' theorem motivates the boundary conditions taken into account.

Lemma 3.1. Let $\Omega \subset \mathbf{R}^{3}$ be as previously defined. Let $f$ be a smooth scalar function defined in $\Omega$. Then $f$ satisfies the following identity:

$$
\begin{aligned}
\int_{\Omega}|\triangle f|^{2}= & \int_{\partial \Omega}\left[\nabla \cdot(\nabla f)(\nu \cdot \nabla f)-\frac{1}{2} \nabla\left(|\nabla f|^{2}\right) \cdot \nu\right] d S \\
& +\sum_{i, j=1,2,3} \int_{\Omega}\left(\partial_{i} \partial_{j} f\right)^{2} d \mathbf{x} .
\end{aligned}
$$

Let $k>q$ be a given constant. We assume that the boundary $\partial \Omega$ satisfies either

$$
\begin{aligned}
& \nabla \omega \cdot \nu=0 \text { on } \Sigma, \quad \nabla \omega \cdot \nu_{i}=k \text { on } \mathcal{S}_{i}, i=1,2, \\
& |\nabla \omega|^{2}=k^{2} \text { on } \Sigma \cup \mathcal{S}_{1} \cup \mathcal{S}_{2},
\end{aligned}
$$

or

$$
\begin{aligned}
& \nabla \omega \cdot \nu= \pm k \text { on } \Sigma, \quad \nabla \omega \cdot \nu_{i}=0 \text { on } \mathcal{S}_{i}, i=1,2, \\
& |\nabla \omega|^{2}=k^{2} \text { on } \Sigma \cup \mathcal{S}_{1} \cup \mathcal{S}_{2} .
\end{aligned}
$$

Such relations correspond to smectic layers reaching the boundary in a perpendicular and tangential fashion, respectively, with a prescribed wave number $k$. In case of (3.7), the surface integration in (3.5) becomes $\pm 2 k^{2} \int_{\Sigma} H d S$, where $H$ is the mean curvature. In constrast, with boundary conditions (3.6) the surface integration in (3.5) is zero.

Remark. The choice of domain and boundary conditions of the problem are motivated by vorticity geometry. Indeed, $\Omega$ and $\Sigma$ play the roles of vortex tube and vortex sheet, respectively. Moreover, $\nabla \omega$ is analogous to the fluid vorticity $\xi$. 


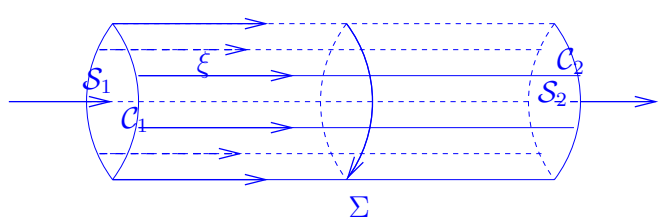

FIG. 3.1. A vortex tube: $\mathcal{C}_{1}$ and $\mathcal{C}_{2}$ are the boundary curves of $\mathcal{S}_{1}$ and $\mathcal{S}_{2}$.

3.2. Existence of minimizers. For simplicity, we restrict ourselves to the case that $\rho$ is constant (say $\rho=1$ ), that is, no nematic defects are present in the sample, and rewrite the smectic energy as follows:

$$
\begin{aligned}
F_{S m}= & D(\Delta \omega-q \nabla \cdot \mathbf{n})^{2}+D\left(|\nabla \omega-q \mathbf{n}|^{2}+\frac{C_{\perp}}{2 D}\right)^{2} \\
& +C_{a}(\nabla \omega \cdot \mathbf{n}-q)^{2}+\left(r+\frac{g}{2}-\frac{C_{\perp}^{2}}{4 D}\right) .
\end{aligned}
$$

We also assume that $\mathbf{D}$ and $\mathbf{E}$ satisfy Maxwell's equations (2.2). We use an electric potential $\varphi$ satisfying $\mathbf{E}=\nabla \varphi$ and impose boundary conditions for $\varphi$ so that (2.2) reads

$$
\left\{\begin{array}{c}
-\nabla \cdot\left(\left(\varepsilon_{\perp} \mathbf{I}+\varepsilon_{a} \mathbf{n} \otimes \mathbf{n}\right) \nabla \varphi\right)=\nabla \cdot \mathbf{P} \text { in } \Omega, \\
\varphi=\varphi_{0} \text { on } \Sigma, \\
-\left(\left(\varepsilon_{\perp} \mathbf{I}+\varepsilon_{a} \mathbf{n} \otimes \mathbf{n}\right) \nabla \varphi\right) \cdot \nu=\mathbf{P} \cdot \nu \text { on } \mathcal{S}_{1} \cup \mathcal{S}_{2},
\end{array}\right.
$$

where $\varphi_{0} \in H^{\frac{1}{2}}(\Sigma)$ is prescribed.

Define

$$
\begin{aligned}
& \mathcal{X}=\left\{(\mathbf{n}, \mathbf{P}) \in W^{1,2}\left(\Omega, \mathbf{S}^{2}\right) \times W^{1,2}\left(\Omega, \mathbf{R}^{3}\right):\|P\|_{\infty} \leq P_{0},\right\} \\
& \mathcal{H}=\left\{\omega \in W^{2,2}(\Omega) \mid \omega \text { satisfies }(3.6) \text { or }(3.7) \text { on } \partial \Omega\right\} \\
& H_{\varphi_{0}}^{1}=\left\{\varphi \in H^{1}(\Omega): \varphi=\varphi_{0} \text { on } \Sigma\right\} \\
& \mathcal{A}^{*}=\mathcal{H} \times \mathcal{X}, \text { and } \\
& \mathcal{A}=\mathcal{A}^{*} \times H_{\varphi_{0}}^{1},
\end{aligned}
$$

where $P_{0}$ is the given polarization saturation constant depending on the material and temperature. For constant potential $\varphi_{0}$, the boundary condition $\varphi=\varphi_{0}$ on $\Sigma$ can be considered as $\varphi=\varphi_{0}$ in $\mathbf{R}^{3}-\Omega$. In this case, the nonlocal energy $\int_{\mathbf{R}^{3}-\Omega}|\mathbf{E}|^{2} d \mathbf{x}$ is zero. For simplicity, we will drop the nonlocal energy in $\mathcal{E}$. We then rewrite the total energy functional $\mathcal{E}$ as a sum:

$$
\mathcal{E}=\mathcal{W}-\frac{1}{2} \int_{\Omega}\left\{\varepsilon_{\perp}|\nabla \varphi|^{2}+\varepsilon_{a}(\mathbf{n} \cdot \nabla \varphi)^{2}+2 \mathbf{P} \cdot \nabla \varphi\right\} d \mathbf{x}
$$

where

$$
\mathcal{W}=\int_{\Omega}\left\{F_{N}+F_{S m}+F_{P}\right\} d \mathbf{x}+\int_{\partial \Omega} \omega_{n}\left(\left(1-\alpha_{0}(\mathbf{n} \cdot \nu)^{2}\right) d S\right.
$$

Since we are interested in the case that $K_{2}$ and $K_{3}$ are large, we assume that $\min \left\{K_{2}, K_{3}\right\} \geq 8 c_{1}[1]$. We note that for all $\mathbf{n} \in \mathbf{W}^{1,2}\left(\Omega, \mathbf{S}^{2}\right)$, the following identities hold:

$$
\begin{aligned}
\operatorname{tr}(\nabla \mathbf{n})^{2} & =|\nabla \mathbf{n}|^{2}-|\nabla \times \mathbf{n}|^{2}, \\
|\nabla \times \mathbf{n}|^{2} & =|\mathbf{n} \cdot \nabla \times \mathbf{n}|^{2}+|\mathbf{n} \times \nabla \times \mathbf{n}|^{2} .
\end{aligned}
$$


Using these identities, we get

$$
\begin{aligned}
F_{N}= & \left(K_{1}-K_{2}-K_{4}\right)(\nabla \cdot \mathbf{n})^{2}+\left(K_{2}+K_{4}\right)|\nabla \mathbf{n}|^{2}-\left(K_{2}+K_{4}\right)|\nabla \times \mathbf{n}|^{2} \\
& +K_{2}(\mathbf{n} \cdot \nabla \times \mathbf{n}+\tau)^{2}+K_{3}|\mathbf{n} \times \nabla \times \mathbf{n}+\mathbf{P}|^{2} .
\end{aligned}
$$

Now, the following inequalities hold:

$$
\begin{aligned}
& K_{2}(\mathbf{n} \cdot \nabla \times \mathbf{n}+\tau)^{2}+K_{3}|\mathbf{n} \times \nabla \times \mathbf{n}-\mathbf{P}|^{2}-\left(K_{2}+K_{4}\right)|\nabla \times \mathbf{n}|^{2} \\
& \geq 4 c_{1}\left(\frac{1}{2}|\mathbf{n} \times \nabla \times \mathbf{n}|^{2}-2|\mathbf{P}|^{2}\right)+2 c_{1}(\mathbf{n} \cdot \nabla \times \mathbf{n}+\tau)^{2}-\left(K_{2}+K_{4}\right)|\nabla \times \mathbf{n}|^{2} \\
& \geq 2 c_{1}|\nabla \times \mathbf{n}+\tau \mathbf{n}|^{2}-8 c_{1}|\mathbf{P}|^{2}-\left(K_{2}+K_{4}\right)|\nabla \times \mathbf{n}|^{2} \\
& \geq 2 c_{1}\left(\frac{1}{2}|\nabla \times \mathbf{n}|^{2}-2 \tau^{2}\right)-8 c_{1}|\mathbf{P}|^{2}-\left(K_{2}+K_{4}\right)|\nabla \times \mathbf{n}|^{2} \\
& \geq\left(c_{1}-K_{2}-K_{4}\right)|\nabla \times \mathbf{n}|^{2}-4 c_{1}\left(\tau^{2}+2|\mathbf{P}|^{2}\right) \\
& \geq-4 c_{1}\left(\tau^{2}+2|\mathbf{P}|^{2}\right) .
\end{aligned}
$$

It follows from (3.12), (3.13), and Lemma 3.1 that $\mathcal{W}$ is bounded below in $\mathcal{A}^{*}$. Therefore, we have

$$
M_{1} \leq \inf _{(\mathbf{n}, \mathbf{P}, \omega) \in \mathcal{A}^{*}} \mathcal{W}(\mathbf{n}, \mathbf{P}, \omega)<\infty
$$

for some $M_{1} \in \mathbf{R}$.

Now, we rewrite the Oseen-frank energy in (3.12) as follows:

$$
\begin{aligned}
F_{N}= & \left(K_{1}-K_{2}-K_{4}\right)(\nabla \cdot \mathbf{n})^{2}+\left(K_{2}+K_{4}\right)|\nabla \mathbf{n}|^{2}-K_{4}\left(\mathbf{n} \cdot \nabla \times \mathbf{n}-\frac{\tau K_{2}}{K_{4}}\right)^{2} \\
& +K\left|\mathbf{n} \times \nabla \times \mathbf{n}+\frac{K_{3}}{K} \mathbf{P}\right|^{2}+\left(K_{3}-\frac{K_{3}^{2}}{K}\right)|\mathbf{P}|^{2}+\tau^{2}\left(K_{2}-\frac{K_{2}^{2}}{\left|K_{4}\right|}\right),
\end{aligned}
$$

where $K=K_{3}-K_{2}-K_{4}$.

Let $\left\{\left(\mathbf{n}^{j}, \mathbf{P}^{j}, \omega^{j}\right)\right\}$ be a minimizing sequence for $\mathcal{W}$. Since $\left|\mathbf{n}^{j}\right|=1$, we get

$$
\begin{aligned}
& \mathbf{n}^{j} \rightarrow \mathbf{n}^{\infty}, \text { in } W^{1,2}(\Omega), \\
& \mathbf{n}^{j} \rightarrow \mathbf{n}^{\infty}, \text { almost everywhere in } \Omega, \quad \text { and } \\
& \mathbf{P}^{j} \rightarrow \mathbf{P}^{\infty} \text { in } W^{1,2}(\Omega) .
\end{aligned}
$$

as $j \rightarrow \infty$. Furthermore, we have

$$
\begin{aligned}
& \mathbf{n}^{j} \times \nabla \times \mathbf{n}^{j} \rightarrow \mathbf{n}^{\infty} \times \nabla \times \mathbf{n}^{\infty} \text { in } L^{2}(\Omega), \\
& \mathbf{n}^{j} \cdot \nabla \times \mathbf{n}^{j} \rightarrow \mathbf{n}^{\infty} \cdot \nabla \times \mathbf{n}^{\infty} \text { in } L^{2}(\Omega),
\end{aligned}
$$

as $j \rightarrow \infty$. Note that for all $j$,

$$
\begin{aligned}
\int_{\Omega}\left|\nabla \omega^{j}\right|^{2} d \mathbf{x} & \leq 2 \int_{\Omega}\left(\left|\nabla \omega^{j}-q \mathbf{n}^{j}\right|^{2}+q^{2}\right) d \mathbf{x}, \quad \text { and } \\
\int_{\Omega}\left|\Delta \omega^{j}\right|^{2} \mathbf{x} & =\int_{\Omega}\left(\left|\Delta \omega^{j}-q \nabla \cdot \mathbf{n}^{j}+q \nabla \cdot \mathbf{n}^{j}\right|^{2} d \mathbf{x}\right. \\
& \leq 2 \int_{\Omega}\left(\left|\Delta \omega^{j}-q \nabla \cdot \mathbf{n}^{j}\right|^{2}+q^{2}\left(\nabla \cdot \mathbf{n}^{j}\right)^{2} d \mathbf{x}\right.
\end{aligned}
$$


hold. From (3.14), (3.15), and Lemma 3.1, we get

$$
\left\|\omega^{j}\right\|_{W^{2,2}(\Omega)} \leq R
$$

for some $R>0$. Hence, we obtain that

$$
\begin{aligned}
& \omega^{j} \rightarrow \omega^{\infty} \text { in } W^{2,2}(\Omega), \quad \text { and } \\
& \nabla \omega^{j} \times \mathbf{n}^{j} \rightarrow \nabla \omega^{\infty} \times \mathbf{n}^{\infty} \text { in } L^{2}(\Omega),
\end{aligned}
$$

as $j \rightarrow \infty$. Using ||$a|-| b|| \leq|a-b|$ for $a$ and $b$ in $\mathbf{R}$, we show that

$$
\left|\left(\left|\nabla \omega^{j} \times \mathbf{n}^{j}\right|\right) \mathbf{P}^{j}-\right| \mathbf{P}^{j}\left|\left(\nabla \omega^{j} \times \mathbf{n}^{j}\right)\right|^{2} \rightarrow\left|\left(\left|\nabla \omega^{\infty} \times \mathbf{n}^{\infty}\right|\right) \mathbf{P}^{\infty}-\right| \mathbf{P}^{\infty}\left|\left(\nabla \omega^{\infty} \times \mathbf{n}^{\infty}\right)\right|^{2}
$$

in $L^{1}$ as $j \rightarrow \infty$.

Since $\mathbf{n} \cdot \nu \in H^{\frac{1}{2}}(\partial \Omega) \subset L^{2}(\partial \Omega)$ with strong topology, $\int_{\partial \Omega} \omega_{n}\left(1-\alpha_{0}(\mathbf{n} \cdot \nu)^{2}\right) d S$ is lower semicontinuous. Therefore, $\mathcal{W}$ is coercive and weakly lower semicontinuous, that is,

$$
\mathcal{W}\left(\mathbf{n}^{\infty}, \mathbf{P}^{\infty}, \omega^{\infty}\right) \leq \lim _{j \rightarrow \infty} \mathcal{W}\left(\mathbf{n}^{j}, \mathbf{P}^{j}, \omega^{j}\right) .
$$

Therefore we have the following lemma.

Lemma 3.2. Assuming that $\min \left\{K_{2}, K_{3}\right\} \geq 8 c_{1}$, there exists a minimizing triple $(\mathbf{n}, \mathbf{P}, \omega)$ of the energy functional $\mathcal{W}$ in $\mathcal{A}^{*}$.

Now, we prove existence of minimizers for $\mathcal{E}$ in $\mathcal{A}$. For any $(\mathbf{n}, \mathbf{P}, \omega) \in \mathcal{A}^{*}$, by the fundamental theory of elliptic PDEs the equation (3.9) has a unique solution which we denote by $\Phi_{\varphi_{0}}(\mathbf{n}, \mathbf{P})$, and thus $\Phi_{\varphi_{0}}(\mathbf{n}, \mathbf{P})$ is the unique minimizer of the functional $-\int_{\Omega}\left(F_{E} d \mathbf{x}\right.$ in $H_{\varphi_{0}}^{1}$. Substituting $\Phi_{\varphi_{0}}(\mathbf{n}, \mathbf{P})$ for $\varphi$ in $\mathcal{E}$, we define $\mathcal{E}^{*}$ by

$$
\mathcal{E}^{*}(\mathbf{n}, \mathbf{P}, \omega)=\mathcal{E}\left(\mathbf{n}, \mathbf{P}, \omega, \Phi_{\varphi_{0}}(\mathbf{n}, \mathbf{P})\right)
$$

After modifying theorem 4.1 in [15], we have the following theorem.

Theorem 3.3. For any $\varphi_{0} \in H^{\frac{1}{2}}(\Sigma)$, there exists a triple $(\mathbf{n}, \mathbf{P}, \omega)$ which minimizes $\mathcal{E}^{*}$ in $\mathcal{A}^{*}$. Furthermore, a quadruple $(\mathbf{n}, \mathbf{P}, \omega, \varphi)$ is a critical point of $\mathcal{E}$, which satisfies (3.9) and

$$
\delta \mathcal{E}(\mathbf{n}, \mathbf{P}, \omega, \varphi)=0 \text { in } \mathcal{A}
$$

if and only if

$$
\varphi=\Phi_{\varphi_{0}}(\mathbf{n}, \mathbf{P}), \text { and } \delta \mathcal{E}^{*}(\mathbf{n}, \mathbf{P}, \omega)=0 \text { in } \mathcal{A}^{*}
$$

TheOREM 3.4. For $\varphi_{0}$ as above and $\min \left\{K_{2}, K_{3}\right\} \geq 8 c_{1}$, there exists a triple $(\mathbf{n}, \mathbf{P}, \omega)$ which minimizes $\mathcal{E}^{*}$ in $\mathcal{A}^{*}$, and therefore $\mathcal{E}$ achieves its minimum in $\mathcal{A}$.

Proof. Let $(\tilde{\mathbf{n}}, \tilde{\mathbf{P}}, \tilde{\omega})$ be a minimizer of $\mathcal{W}$ in $\mathcal{A}^{*}$. Then

$$
\inf _{(\mathbf{n}, \mathbf{P}, \omega) \in \mathcal{A}^{*}} \mathcal{E}^{*}(\mathbf{n}, \mathbf{P}, \omega) \leq \mathcal{E}^{*}(\tilde{\mathbf{n}}, \tilde{\mathbf{P}}, \tilde{\omega})<\infty .
$$

Let

$$
A(\nabla \varphi, \mathbf{n})=\left[\left(\varepsilon_{\perp} \mathbf{I}+\varepsilon_{a} \mathbf{n} \otimes \mathbf{n}\right) \nabla \varphi\right] \cdot \nabla \varphi .
$$


If $\tilde{\varphi}$ is some fixed $W^{1,2}$ extension of $\varphi_{0}$ to $\Omega$, then for any $\eta>0$,

$$
\begin{aligned}
-2 \int_{\Omega} F_{E} d \mathbf{x} & =\int_{\Omega}\left[A\left(\nabla \Phi_{\varphi_{0}}(\mathbf{n}, \mathbf{P}), \mathbf{n}\right)+\mathbf{P} \cdot \nabla \Phi_{\varphi_{0}}(\mathbf{n}, \mathbf{P})\right] d \mathbf{x} \\
& \leq \int_{\Omega}[A(\nabla \tilde{\varphi}, \mathbf{n})+\mathbf{P} \cdot \nabla \tilde{\varphi}] d \mathbf{x} \\
& \leq C\left(\Omega, \varphi_{0}\right),
\end{aligned}
$$

for some $C\left(\Omega, \varphi_{0}\right)$ depending on $\Omega$ and $\varphi_{0}$. Since $\mathcal{W}$ is bounded from below, it follows from the above that $\mathcal{E}^{*}$ is also bounded below.

Now, choose a minimizing sequence $\left(\mathbf{n}^{i}, \mathbf{P}^{i}, \omega^{i}\right)$ in $\mathcal{A}^{*}$ and set $\varphi^{i}=\Phi_{\varphi_{0}}\left(\mathbf{n}^{i}, \mathbf{P}^{i}\right)$. Using the same computation as in the proof of the previous lemma, we obtain that

$$
\begin{aligned}
& \mathbf{n}^{j} \rightarrow \mathbf{n}^{\infty} \text { in } W^{1,2}, \\
& \mathbf{n}^{j} \rightarrow \mathbf{n}^{\infty} \text { almost everywhere in } \Omega, \\
& \mathbf{P}^{j} \rightarrow \mathbf{P}^{\infty} \text { in } W^{1,2}, \quad \text { and } \\
& \omega^{j} \rightarrow \omega^{\infty} \text { in } W^{2,2}
\end{aligned}
$$

as $j \rightarrow \infty$. Since $\left(\mathbf{P}^{j}\right)$ converges strongly to $\mathbf{P}^{\infty}$ in $L^{2}$,

$$
\int_{\Omega} \mathbf{P}^{j} \cdot \nabla \xi d \mathbf{x} \rightarrow \int_{\Omega} \mathbf{P}^{\infty} \cdot \nabla \xi d \mathbf{x} \text { as } j \rightarrow \infty
$$

for any $\xi \in H^{1}(\Omega)$. It follows from (3.9) and (3.18) that $\left(\varphi^{j}\right)$ converges strongly to $\varphi^{\infty}$ in $H_{\varphi_{0}}^{1}$. have

Since $\varphi^{i}$ is the minimizer of $-\int_{\Omega} F_{E}\left(\mathbf{n}^{i}, \mathbf{P}^{i}, \nabla \varphi\right) d \mathbf{x}$ for each fixed $\mathbf{n}^{i}$ and $\mathbf{P}^{i}$, we

$$
-\int_{\Omega} F_{E}\left(\mathbf{n}^{i}, \mathbf{P}^{i}, \nabla \varphi^{i}\right) d \mathbf{x} \leq-\int_{\Omega} F_{E}\left(\mathbf{n}^{i}, \mathbf{P}^{i}, \nabla \varphi^{\infty}\right) d \mathbf{x} .
$$

By Lebesgue's theorem, we obtain

$$
\lim _{i \rightarrow \infty}\left\{-\int_{\Omega} F_{E}\left(\mathbf{n}^{i}, \mathbf{P}^{i}, \nabla \varphi^{i}\right) d \mathbf{x}\right\}=-\int_{\Omega} F_{E}\left(\mathbf{n}^{\infty}, \mathbf{P}^{\infty}, \nabla \varphi^{\infty}\right) d \mathbf{x},
$$

so that

$$
\limsup _{i \rightarrow \infty}\left\{-\int_{\Omega} F_{E}\left(\mathbf{n}^{i}, \mathbf{P}^{i}, \nabla \varphi^{i}\right) d \mathbf{x}\right\} \leq-\int_{\Omega} F_{E}\left(\mathbf{n}^{\infty}, \mathbf{P}^{\infty}, \nabla \varphi^{\infty}\right) d \mathbf{x} .
$$

This implies that

$$
\liminf _{i \rightarrow \infty} \int_{\Omega} F_{E}\left(\mathbf{n}^{i}, \mathbf{P}^{i}, \nabla \varphi^{i}\right) d \mathbf{x} \geq \int_{\Omega} F_{E}\left(\mathbf{n}^{\infty}, \mathbf{P}^{\infty}, \nabla \varphi^{\infty}\right) d \mathbf{x} .
$$

Since $\mathcal{W}$ is lower semicontinuous, we finally conclude that

$$
\mathcal{E}^{*}\left(\mathbf{n}^{\infty}, \mathbf{P}^{\infty}, \omega^{\infty}\right)=\inf _{(\mathbf{n}, \mathbf{P}, \omega) \in \mathcal{A}^{*}} \mathcal{E}^{*}(\mathbf{n}, \mathbf{P}, \omega)
$$

$\square$ 
3.3. Applications. Problems analogous to the model problem of the previous subsection are often found in applications. Let us discuss two examples. First we consider the case of a liquid crystal contained in a dielectric liquid media with free ions that form a charged layer of density $\sigma$ on the interface. Letting $\Omega$ be a bounded domain occupied by liquid crystals, the goal is to find an electric potential $\varphi \in W^{1,2}\left(\mathbf{R}^{3}\right)$ satisfying

$$
\left\{\begin{array}{c}
-\nabla \cdot\left(\left(\varepsilon_{\perp} \mathbf{I}+\varepsilon_{a} \mathbf{n} \otimes \mathbf{n}\right) \nabla \varphi\right)=\nabla \cdot \mathbf{P} \text { in } \Omega \\
-\Delta \varphi=0 \text { in } \mathbf{R}^{3}-\bar{\Omega} \\
-\left[\left(\varepsilon_{\perp} \mathbf{I}+\varepsilon_{a} \mathbf{n} \otimes \mathbf{n}\right) \nabla \varphi-\varepsilon_{0} \nabla \varphi\right] \cdot \nu=\mathbf{P} \cdot \nu+\sigma \text { on } \partial \Omega
\end{array}\right.
$$

where $\varepsilon_{0}$ is the dielectric coefficient of the media.

The second case comes up in device applications. Now the liquid crystal domain $\Omega$ is confined between two conducting plates $\Omega_{1}$ and $\Omega_{2}$ respectively. We assume that there is no free charge in $\Omega$, and we neglect end-effects. In this case, the boundary value problem is stated as follows: For a given potential $\tilde{\varphi}$, find $\varphi$ satisfying the following conditions:

$$
\left\{\begin{array}{c}
-\nabla \cdot\left(\left(\varepsilon_{\perp} \mathbf{I}+\varepsilon_{a} \mathbf{n} \otimes \mathbf{n}\right) \nabla \varphi\right)=\nabla \cdot \mathbf{P} \text { in } \Omega, \\
-\Delta \varphi=0 \text { in } \mathbf{R}^{3}-\left(\bar{\Omega} \cup \bar{\Omega}_{1} \cup \bar{\Omega}_{2}\right), \\
\left(\varepsilon_{\perp} \nabla \varphi+\varepsilon_{a}(\mathbf{n} \cdot \nabla \varphi) \mathbf{n}+\mathbf{P}\right) \cdot \nu=0 \text { on } \partial \Omega_{1} \cup \partial \Omega_{2}, \\
\nabla \varphi=\mathbf{0} \text { in } \Omega_{1}, \quad \varphi=\tilde{\varphi} \text { in } \Omega_{2}, \\
\varphi \rightarrow 0 \text { as }|\mathbf{x}| \rightarrow \infty
\end{array}\right.
$$

If $\tilde{\varphi}$ is set to zero, $\nabla \varphi$ gives the electric field created by the polar distribution. Analogous boundary value problems for ferroelectric solids are considered in [35]. The existence proofs developed in the previous section extend with some modifications to the present examples.

3.4. Shapes and polarization. We close this section with examples to illustrate the relationship between the nonlocal energy and the shape of the domain.

Example 1. Uniformly polarized rectilinear cylinder. Let $\Omega$ be a cylinder in $\mathbf{R}^{3}, x^{2}+y^{2} \leq r_{1}^{2}$, occupied by a smectic $\mathrm{C}$ material such that

$$
\nabla \omega=\mathbf{e}_{r}, \quad \mathbf{n}=f(r) \mathbf{e}_{r}+g(r) \mathbf{e}_{\theta}, \quad \mathbf{P}=P_{0} \mathbf{k} .
$$

Let $\tilde{\Omega}$ denote a second cylinder, $x^{2}+y^{2} \leq r_{2}^{2}, r_{1}<r_{2}$. Suppose that $\mathbf{E}$ is the electric field on the cylindrical surface $\partial \tilde{\Omega}$. Applying Gauss' theorem to $\tilde{\Omega}$, we get

$$
\int_{\partial \tilde{\Omega}} \mathbf{E} \cdot \nu d S=0
$$

since the net charge inside $\tilde{\Omega}$ is zero. By symmetry, we observe that $|\mathbf{E}|$ is constant on $\partial \tilde{\Omega}, \mathbf{E} \cdot \nu=|\mathbf{E}|$, and thus

$$
\int_{\mathbf{R}^{3}-\Omega}|\mathbf{E}|^{2} d \mathbf{x}=0
$$

We note that the electric field $\mathbf{E}$ due to the polarization may not be zero outside $\Omega$ if the shape is non-symmetric. For instance, in the case of a bent cylindrical domain, the electric field created by the polarization is not rotationally symmetric. Therefore, 
we cannot conclude that $|\mathbf{E}|$ is constant on $\partial \tilde{\Omega}$, and so $\mathbf{E}$ is, in general, non-zero on $\partial \tilde{\Omega}$. In this case, the self energy $\int_{\mathbf{R}^{3}-\Omega}|\mathbf{E}|^{2} d \mathbf{x}$ is also non-zero.

Example 2. Polarized helical filament. In example 1, we replace $\Omega$ by a thin filament [2],

$$
\Omega=\left\{\mathbf{x} \in \mathbf{R}^{3}: \mathbf{x}=\mathcal{C}(s)+\xi \mathbf{e}, s \in[0, l], \xi \in[0, r], \mathbf{e} \cdot \mathbf{T}=0, \mathbf{e} \cdot \mathbf{e}=1\right\}
$$

where $\mathcal{C}:[0, l] \rightarrow \mathbf{R}^{3}$ is a smooth curve and $\mathbf{T}$ is the unit tangent vector. The domain $\Omega$ is a thin filament and not necessarily a right cylinder. Let $\mathbf{N}$ and $\mathbf{B}$ denote the normal and binormal vectors to the curve, respectively.

The curve $\mathcal{C}$ represents the center curve of the curvilinear cylindrical domain $\Omega$. We assume that for each $s \in[0, r]$, the smectic layer normal is parallel to $\mathbf{T}$, and the director field $\mathbf{n}$ is parallel to the plane determined by $\mathbf{T}$ and $\mathbf{B}$, making a constant tilt angle with T. Accordingly, we set

$$
\nabla \omega=\mathbf{T}, \quad \mathbf{n}=\alpha \mathbf{T}+\beta \mathbf{B}, \alpha^{2}+\beta^{2}=1, \quad \mathbf{P}=-P_{0} \mathbf{N} .
$$

Define a coordinate system $(s, \xi, \theta)$ so that

$$
\mathbf{e}_{\xi}=\cos \theta \mathbf{N}+\sin \theta \mathbf{B}, \quad \mathbf{e}_{\theta}=-\sin \theta \mathbf{N}+\cos \theta \mathbf{B} .
$$

Since the net charge in $\tilde{\Omega}$ is zero, by Gauss' theorem

$$
\int_{\partial \tilde{\Omega}} \mathbf{E} \cdot \nu d S=0
$$

In general, though, we cannot conclude that $\mathbf{E}$ is symmetric around the curve $\mathcal{C}$, and so $\mathbf{E}$ may not be zero outside the filament region. Now the question is whether or not there is a shape such that $\mathbf{E}=\mathbf{0}$ in $\mathbf{R}^{3}-\Omega$. Heuristically, we can view such a shape as the limiting case of a helical filament as the pitch approaches zero. Note that this would allow us to recover the symmetry property of the domain and of the electric field, and conclude that $\int_{\mathbf{R}^{3}-\Omega}|\mathbf{E}|^{2}$ is negligible.

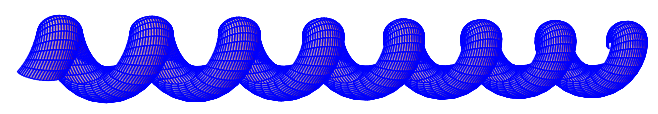

FIG. 3.2. A polarized helical filament

4. Classification of energy minimizers. We apply asymptotic arguments to determine whether energy minimizers correspond to either helical configurations or ferroelectric ones. In this section, we consider the energy as in (2.14). We wish to identify the smectic layer geometry and find parameter conditions leading to helical director configurations in the bulk with zero average polarization, as well as those giving homogeneous ferroelectric states. For this, we will consider a rectangular domain between two parallel plates:

$$
\Omega=\{\mathbf{x}=(x, y, z): 0<y, z<L, 0<x<d .\},
$$

for fixed $0<L, 0<d$. Let $\mathbf{i}, \mathbf{j}$ and $\mathbf{k}$ denote the corresponding orthonormal system of vectors. 
4.1. Helical energy minimizers. We determine the structure of the energy minimizers $(\mathbf{n}, \omega, \mathbf{P})$ when $K_{2}$ and $K_{3}$ as well as the smectic coefficients dominate over the Ginzburg-Landau energy and surface energy parameters, and $C_{\perp}<0$. Such a situation arises at temperatures below the threshold of the smectic $\mathrm{A}$ to smectic $\mathrm{C}$ transition yielding helical configurations of $\mathbf{n}$ and $\mathbf{P}$. It is well known that in the higher temperature transition from nematic to smectic $\mathrm{A}, K_{2}$ becomes unbounded and the smectic coefficient $C_{\perp} \geq 0$.

We take the admissible set so that

$$
k=q \sqrt{\frac{\left|C_{\perp}\right|}{2 D q^{2}}+1} .
$$

We consider admissible fields such that $\mathbf{n}$ makes a constant angle $\alpha$ with the layer normal vector $\nabla \omega$. We also choose $\alpha$ such that

$$
\tan \alpha=\sqrt{\frac{\left|C_{\perp}\right|}{2 D q^{2}}} .
$$

Specifically, we let

$$
\left\{\begin{array}{l}
\mathbf{n}_{0}=\left(a \cos \frac{\tau z}{a^{2}}, a \sin \frac{\tau z}{a^{2}}, c\right), \\
a=\sin \alpha \neq 0, \quad \frac{a^{2}}{c^{2}}=\frac{\left|C_{\perp}\right|}{2 D q^{2}} \\
\mathbf{P}_{0}=\frac{c \tau}{a}\left(-\sin \frac{\tau z}{a^{2}} \mathbf{i}+\cos \frac{\tau z}{a^{2}} \mathbf{j}\right), \\
\omega_{0}=k z, \quad k=\frac{q}{c}, \quad \nu=\mathbf{i} .
\end{array}\right.
$$

A simple calculation gives

$$
\begin{aligned}
& \nabla \omega_{0} \cdot \mathbf{n}_{0}=q, \quad \triangle \omega_{0}=0, \quad\left|\nabla \omega_{0}-q \mathbf{n}_{0}\right|^{2}=\frac{\left|C_{\perp}\right|}{2 D}, \\
& \nabla \cdot \mathbf{n}_{0}=0, \quad \mathbf{n}_{0} \cdot \nabla \times \mathbf{n}_{0}+\tau=0, \quad\left|\mathbf{n}_{0} \times\left(\nabla \times \mathbf{n}_{0}\right)+\mathbf{P}_{0}\right|=0, \\
& \nabla \cdot \mathbf{P}_{0}=0, \quad\left|\nabla \mathbf{P}_{0}\right|=\frac{c \tau^{2}}{a^{3}}, \quad \mathbf{P}_{0}=\nabla \omega_{0} \times \mathbf{n}_{0} .
\end{aligned}
$$

We observe that the quantity $\tan \alpha=\sqrt{\frac{\left|C_{\perp}\right|}{2 D q^{2}}}$ is of the order of $\tan \frac{\pi}{6}$ according to experimental measurements of the director tilt angle. This together with available information on the wave number $q$ in the smectic A phase allows us to determine the relative value of the smectic parameters $\left|C_{\perp}\right|$ and $D$.

The total energy corresponding to the fields in (4.3) is given by

$$
\begin{aligned}
\mathcal{E}_{0}= & L^{2} d\left[r+\frac{g}{2}-\frac{C_{\perp}^{2}}{4 D}+\left(K_{2}+K_{4}\right) \frac{\tau^{2}}{a^{2}}+\frac{c^{2} \tau^{2}}{a^{2}}\left(B \frac{\tau^{2}}{a^{4}}+a_{0}+b_{0} \frac{c^{2}}{a^{2}} \tau^{2}\right)\right] \\
& +\left(2 L^{2}+4 d L\right)\left(C_{p} \omega_{p}+C_{r} \omega_{r}+C_{n} \omega_{n}\right)
\end{aligned}
$$

where $C_{r}, C_{p}, C_{n}$ are expressions involving $a, c, q$ and $\tau$.

Letting $K_{0}=\frac{1}{2} \min \left\{K_{2}, K_{3}\right\}$, we write the Oseen-Frank energy as follows:

$$
\begin{aligned}
F_{N} & =\left(K_{2}-K_{0}\right)|\mathbf{n} \cdot \nabla \times \mathbf{n}+\tau|^{2}+\left(K_{3}-K_{0}\right) \mathbf{n} \times \nabla \times \mathbf{n}+\left.\mathbf{P}\right|^{2} \\
& +\left(K_{1}-K_{2}-K_{4}\right)(\nabla \cdot \mathbf{n})^{2}+\left(K_{2}+K_{4}\right)|\nabla \mathbf{n}|^{2} \\
& +K_{0}|\mathbf{n} \cdot \nabla \times \mathbf{n}+\tau|^{2}+K_{0}|\mathbf{n} \times \nabla \times \mathbf{n}+\mathbf{P}|^{2}-\left(K_{2}+K_{4}\right)|\nabla \times \mathbf{n}|^{2} .
\end{aligned}
$$


We invoke the following estimate for the last three terms

$$
\begin{aligned}
& K_{0}\left(|\mathbf{n} \cdot \nabla \times \mathbf{n}+\tau|^{2}+|\mathbf{n} \times \nabla \times \mathbf{n}+\mathbf{P}|^{2}\right)-\left(K_{2}+K_{4}\right)|\nabla \times \mathbf{n}|^{2} \\
& \geq-4 c_{1}\left(\tau^{2}+2|\mathbf{P}|^{2}\right) .
\end{aligned}
$$

We then obtain the following estimates:

$$
\begin{aligned}
& \int_{\partial \Omega} G_{S} d S \geq-\left(2\left|\omega_{p}\right|+2\left|\omega_{r}\right|+\left|\omega_{n}\right|(1+|\alpha|)\left(2 L^{2}+4 d L\right),\right. \\
& \int_{\Omega}\left(F_{S m}+F_{N}+G_{P}\right) d \mathbf{x} \geq\left[r+\frac{g}{2}-\left(\frac{C_{\perp}^{2}}{4 D}+\frac{\left(a_{0}-8 c_{1}\right)^{2}}{4 b_{0}}+4 c_{1} \tau^{2}\right)\right] d L^{2} .
\end{aligned}
$$

Letting $(\mathbf{n}, \mathbf{P}, \omega)$ denote an energy minimizer, we get

$$
\begin{aligned}
0 \leq & \mathcal{E}(\mathbf{n}, \mathbf{P}, \omega)+\left(2\left|\omega_{p}\right|+2\left|\omega_{r}\right|+\left|\omega_{n}\right|(1+|\alpha|)\left(2 L^{2}+4 d L\right)\right. \\
& +\left[\frac{C_{\perp}^{2}}{4 D}+\frac{\left(a_{0}-8 c_{1}\right)^{2}}{4 b_{0}}+4 c_{1} \tau^{2}-r-\frac{g}{2}\right] d L^{2} . \\
\leq & \mathcal{E}_{0}+\left(2\left|\omega_{p}\right|+2\left|\omega_{r}\right|+\left|\omega_{n}\right|(1+|\alpha|)\left(2 L^{2}+4 d L\right)\right. \\
& +\left[\frac{C_{\perp}^{2}}{4 D}+\frac{\left(a_{0}-4 c_{1}\right)^{2}}{4 b_{0}}+4 c_{1} \tau^{2}-r-\frac{g}{2}\right] d L^{2} \equiv \overline{\mathcal{E}_{0}} .
\end{aligned}
$$

Since $\frac{\left|C_{\perp}\right|}{2 D q^{2}}$ is bounded, we note that the quantity on the right hand side of the inequality is independent of $D, C_{\perp}, K_{1}, K_{2}$ and $K_{3}$, with the only $K_{i}$ constants appearing as the sum $K_{2}+K_{4}$. From (4.5) together with (3.12) and (3.13), we get the following theorem:

TheOREm 4.1. Let $q>0, \tau>0$ be fixed. Suppose that the constitutive parameters satisfy assumptions (3.1)-(3.3). Suppose that $K_{2}, K_{3} \geq 8 c_{1}$ and $0<\frac{\left|C_{\perp}\right|}{2 D q^{2}} \leq 1$. If $(\mathbf{n}, \mathbf{P}, \omega)$ is a minimizer of $\mathcal{E}$, then the following estimates hold:

$$
\begin{aligned}
& \|(\nabla \omega \times \mathbf{n})|\mathbf{P}|-\mathbf{P}(|\nabla \omega \times \mathbf{n}|)\|_{2, \Omega}^{2} \leq \epsilon^{2} \overline{\mathcal{E}}_{0} \\
& \|\nabla \mathbf{n}\|_{2, \Omega} \leq \frac{\overline{\mathcal{E}}_{0}}{K_{2}+K_{4}}, \\
& \|\mathbf{n} \times \nabla \times \mathbf{n}+\mathbf{P}\|_{2, \Omega}^{2} \leq \frac{\overline{\mathcal{E}_{0}}}{\min \left\{K_{2}, K_{3}\right\}} \\
& \|\mathbf{n} \cdot \nabla \times \mathbf{n}+\tau\|_{2, \Omega}^{2} \leq \frac{\overline{\mathcal{E}_{0}}}{\min \left\{K_{2}, K_{3}\right\}} \\
& \left\|\left|\frac{1}{q} \nabla \omega-\mathbf{n}\right|^{2}-\frac{\left|C_{\perp}\right|}{2 D q^{2}}\right\|_{2, \Omega}^{2} \leq \frac{\overline{\mathcal{E}}_{0}}{D q^{2}} \\
& \left\|\frac{1}{q} \nabla \omega \cdot \mathbf{n}-1\right\|_{2, \Omega}^{2} \leq \frac{\overline{\mathcal{E}}_{0}}{C_{a}} \\
& \|\nabla \mathbf{P}\|_{2, \Omega} \leq \frac{\overline{\mathcal{E}}_{0}}{B}
\end{aligned}
$$

Next, we proceed to take limits in (4.6)-(4.11). We use the following representation for $\mathbf{n}$ :

$$
\mathbf{n}=\sin \theta \cos \phi \mathbf{i}+\sin \theta \sin \phi \mathbf{j}+\cos \theta \mathbf{k},
$$

where $\phi=\phi(x, y, z)$ and $\theta=\theta(x, y, z)$ are functions resulting from energy minimization. 
TheOREm 4.2. Suppose that the hypotheses of the previous theorem hold. Then the energy minimizing fields $(\mathbf{n}, \mathbf{P}, \omega)$ satisfy the following limiting relations:

$$
\begin{aligned}
& \lim _{C_{a} \rightarrow \infty} \nabla \omega \cdot \mathbf{n}=q, \\
& \lim _{\left|C_{\perp}\right| \rightarrow \infty}|\nabla \omega|=q \sqrt{\frac{\left|C_{\perp}\right|}{2 D q^{2}}+1,} \\
& \lim _{\epsilon \rightarrow 0} \mathbf{P}=\cot \alpha \tau \frac{\mathbf{k} \times \mathbf{n}}{|\mathbf{k} \times \mathbf{n}|}, \quad \cot \alpha=\sqrt{\frac{2 D q^{2}}{\left|C_{\perp}\right|},} \\
& \lim _{K \rightarrow \infty}(\mathbf{n} \times \nabla \times \mathbf{n}+\mathbf{P})=0, \\
& \lim _{K \rightarrow \infty}(\mathbf{n} \cdot \nabla \times \mathbf{n}+\tau)=0,
\end{aligned}
$$

where $K=\min \left\{K_{2}, K_{3}\right\}$. Furthermore, we get

$$
\begin{aligned}
& \lim _{\left|C_{\perp}\right| \rightarrow \infty} \omega=\left(q \sqrt{\frac{\left|C_{\perp}\right|}{2 D q^{2}}+1}\right) z, \\
& \lim _{K \rightarrow \infty} \mathbf{n}=\sin \alpha \cos \frac{\tau}{a^{2}} z \mathbf{i}+\sin \alpha \sin \frac{\tau}{a^{2}} z \mathbf{j}+\cos \alpha \mathbf{k} .
\end{aligned}
$$

Proof. From the geometry of the domain and the boundary conditions, it follows that $\nabla \omega=|\nabla \omega| \mathbf{k}$, which together with (4.10) and (4.11) yield (4.13), (4.14), and (4.18). It now follows from (4.13) and (4.14) that $\theta=\alpha$ is the constant given by (4.2). These together with (4.6) yield $\mathbf{P}=|\mathbf{P}| \frac{\mathbf{k} \times \mathbf{n}}{|\mathbf{k} \times \mathbf{n}|}$. Combining this equation with (4.16) and (4.17) gives $\phi=\frac{\tau}{a^{2}} z$ in (4.19). This also yields (4.15). $\square$

Note that from the property $\lim _{D \rightarrow \infty}(\triangle \omega-q \nabla \cdot \mathbf{n})=0$, it follows that the limiting director field has zero divergence, in agreement with (4.19).

4.2. Ferroelectric energy minimizers. In the previous theorem, the elasticity constants $K_{2}$ and $K_{3}$ become unbounded with respect to the parameters of the polarization contribution to the energy. We will show that the ferroelectric configurations,

$$
\mathbf{n}= \pm \sin \alpha \mathbf{j}+\cos \alpha \mathbf{k}, \quad \mathbf{P}= \pm P_{0} \mathbf{i}, \quad P_{0}=\sqrt{\frac{\left|a_{0}\right|}{b_{0}}}
$$

with $\alpha$ the constant in (4.15), are limits of minimizers at the limit of $K_{1}$ large, and when the polar coefficients $\omega_{p}$ and $\omega_{r}$ dominate over the twist and bending elasticity constants $K_{2}$ and $K_{3}$. This situation occurs at temperatures lower than those of the helical regime. The role of the surface energy is also relevant in such a case.

Next, we take the following set of admissible fields to determine the ferroelectric limits:

$$
\mathbf{n}= \pm \sin \alpha \mathbf{j}+\cos \alpha \mathbf{k}, \quad \mathbf{P}= \pm \sqrt{\frac{\left|a_{0}\right|}{b_{0}} \mathbf{i}}
$$

with $0<\alpha<\frac{\pi}{2}$, and $\omega$ as in (4.15) and (4.18), respectively. We find that the energy $\mathcal{E}_{1}$ corresponding to such fields is:

$$
\begin{aligned}
\mathcal{E}_{1}=L^{2} & {\left[\left(K_{2} \tau^{2}+\frac{\left|a_{0}\right|}{b_{0}} K_{3}\right) d+2\left[\omega_{p}+\omega_{r}+\omega_{n}-\left(\frac{\left|a_{0}\right|}{b_{0}}\right)^{2} \omega_{r}\right.\right.} \\
& \left.\left.-\alpha_{0} \omega_{n} \sin ^{2} \alpha\right)\right] .
\end{aligned}
$$


Replacing $\mathcal{E}_{0}$ with $\mathcal{E}_{1}$, the estimates of Theorem 4.1 hold. These allow us to establish the following asymptotic limits of minimizers:

$$
\begin{array}{lll}
\nabla \cdot \mathbf{n}=0 & \text { as } & K_{1} \rightarrow \infty, \\
|\nabla \omega|=k & \text { as } & \left|C_{\perp}\right| \rightarrow \infty .
\end{array}
$$

Letting $D \rightarrow \infty$ and taking (4.23) into account, it follows that $\triangle \omega=0$. This together with the boundary conditions on $\partial \Omega$ gives $\nabla \omega=(0,0, k)$, with $k$ as in (4.1). Moreover, letting $C_{a} \rightarrow \infty$ gives $\cos \alpha=\frac{q}{k}$, and $\mathbf{P}=\sqrt{\frac{\left|a_{0}\right|}{b_{0} \mid}} \frac{\mathbf{k} \times \mathbf{n}}{|\mathbf{k} \times \mathbf{n}|}$ results by letting $\epsilon \rightarrow 0$ and using the expression for $\nabla \omega$. By letting $\omega_{r} \rightarrow \infty$, we get $\phi= \pm \frac{\pi}{2}$.

We finally make the following remarks:

1. The limiting fields $(\mathbf{n}, \mathbf{P}, \omega)$ given by (4.18) and (4.20) satisfy the Euler-Lagrange equations with the prescribed boundary conditions.

2. Likewise, $(\mathbf{n}, \mathbf{P}, \omega)$ as in (4.18) and (4.19) solve the Euler-Lagrange equations at the limit $\left|C_{\perp}\right| \rightarrow \infty$.

5. Conclusions. We studied modeling of ferroelectirc smectic $\mathrm{C}^{*}$ liquid crystals and investigated nonlocal electrostatic effects. We discussed how the proposed model is consistent with well-known approaches found in the physics literature. We proved existence of minimizers for the total energy by means of direct methods of calculus of variations, within the class of fields satisfying physically relevant boundary conditions, with respect to the layering configuration. We presented examples to illustrate the relationship between domain shape and reduction of the nonlocal energy. For instance, we argued that a thin filament may become helical in order to lower the nonlocal energy. We also studied the asymptotic properties of the energy minimizers as the parameters of the energy become unbounded upon the temperature reaching transition values from smectic $\mathrm{C}^{*}$ to lower temperature ferroelectric limits.

6. Acknowledgement. We would like to thank professor Marta Lewicka for many interesting comments and suggestions.

\section{REFERENCES}

[1] P. Bauman, M. C. Calderer, C. Liu, and D. Phillips, The phase transition between Chiral and Smectic A* liquid crystals, Arch. Ration. Mech. Anal., 165 (2002), pp. 161-186.

[2] P. Biscari And M. C. CALDerer, Telephone-cord instabilities in thin smectic capillaries, Phys. Rev. E, 71 (2005), p. 051701.

[3] J. Chen And T. C. Lubensky, Landau-Ginzburg mean-field theory for the nematic to smectic C and nematic to smectic A liquid crystal transistions, Phys. Rev. A, 14 (1976), pp. 12021297.

[4] N. A. Clark and S. T. Lagerwall, Submicrosecond bistable electro-optic switching in liquid crystals, Appl. Phys. Lett., 36 (1980), pp. 899-901.

[5] D. A. Coleman, J. Fernsler, N. Chattham, M. Nakata, Y. Takanishi, E. Köblova, D. R. Link, R. F. Shao, W. G. Jang, J. E. Maclennan, O. Mondainn-Monval, C. Boyer, W. Weissflog, G. Petzel, L. C. Chien, J. Zasadzinski, J. Watanabe, D. M. Walba, H. Takezoe, and N. A. Clark, Polarization-Modulated liquid crystal phases, Science, 301 (2003), pp. 1204-1211.

[6] P. J. Collings and J. S. Patel, eds., Handbook of Liquid Crystal Research, Oxford University Press, New York, 1997.

[7] P. G. De Gennes and J. Prost, The Physics of Liquid Crystals, Clarendon Press, 1993.

[8] Z. Dogic and S. Fraden, Development of model colloidal liquid isotropic-smectic transition, Phil. Trans. R. Soc. Lond. A, 359 (2001), pp. 997-1015.

[9] F. C. Frank, On the theory of liquid crystals, Discuss. Faraday Soc., 25 (1958), pp. 19-28.

[10] M. Glogarova, J. Fousek, L. Lejcek, and J. Pavel, The structure of ferroelectric liquid crystals in planar and its response to electric fields, Ferroelectrics, 58 (1984), pp. 161-178. 
[11] M. Glogarova, L. Lejcek, and J. Pavel, The influence of an external electric field on the structure of chrial Sm $C^{*}$ liquid crystals, Mol. Cryst. Liq. Cryst., 91 (1983), pp. 309-325.

[12] M. Glogarova And J. Pavel, The structure of chiral Sm $C^{*}$ liquid crystals in planar samples and its change in an electric field, J. Physique, 45 (1984), pp. 143-149.

[13] J. W. Goody And ET AL, Ferroelectric Liquid Crystals, principles, properties and applications, Gordon and Breach Science Publishers, 1991.

[14] M. A. Handschy And N. A. Clark, Structures and responses of ferroelectric liquid crystals in the syrface-stabilized geometry, Ferroelectrics, 59 (1984), pp. 69-116.

[15] R. Hardt, D. Kinderlehrer, And F. H. Lin, Existence and partial regularity of static liquid crystal configurations, Comm. Math. Phys., 105 (1986), pp. 547-570.

[16] A. Jákli, Ch. Lischka, W. Weissflog, G. Pelzl, and A. Saupe, Helical filamentary growth in liquid crystals consisting of banana-shaped molecules, Liq. Cryst., 27 (2000), pp. 14051409.

[17] A. Jákli, D. Krüerke, And G. G. NAIR, Liquid crystal fibers of bent-core molecules, Phys. Rev. E, 67 (2003), p. 051702.

[18] S. Joo And D. Phillips, Phase transitions between chiral nematic, smectic $A^{*}$, and $C^{*}$ liquid crystals. Preprint, 2004.

[19] A. G. Khachaturyan, Development of helical cholesteric structure in a nematic liquid crystal due to the dipole-dipole interaction, J. Phys. Chem. Solids, 36 (1975), pp. 1055-1061.

[20] X. Y. Kong AND Z. L. WAng, Polar-surface dominated $\mathrm{ZnO}$ nanobelts and the elctrostatic energy induced nanohelixes, nanosprings, and nanospirals, Appl. Phys. Letters, 84 (2004), pp. $975-977$.

[21] S. T. Lagerwall, Ferroelectric and Antiferroelectric Liquid Crystals, Wiley-VCH, 1999.

[22] L. D. Landau, E. M. Lifshitz, and L. P. Pitaevskil, Electrodynamics of Continuous Media, Butterworth-Heinemann, Oxford, 1998.

[23] L. Longa, D. Monselesan, and H. R. Trebin, An extension of the Landau-Ginzburgde Gennes theory for liquid crystals, Liq. Cryst., 2 (1987), pp. 769-796.

[24] I. LuKYanchuk, Phase transition between the cholesteric and twist grain boundary $C$ phases, Phys. Rev. E, 57 (1998), pp. 574-581.

[25] R. B. Meyer, L. Liebert, L. Strzelecki, and P. Keller, , Ferroelectric liquid crystals, J. de Physique, Lettres, 36(1975), pp. L69-L71.

[26] I. MušEvič, R. Blinc, AND B. ŽEKš, The Physics of Ferroelectric and Antiferroelectric Liquid Crystals, World-Scientific, Singapore, New Jersey, London, Hong Kong, 2000.

[27] J. PARK AND M. C. CALDERER, Variational probelms and modeling of ferroelectricity in chiral smectic liquid crystals, The IMA volumes in Mathematics and Its applications, 141 (2005), pp. 169-188.

[28] J. PARK AND M. C. CALDERER, Phase transitions between ferroelectric and antiferroelectric liquid crystal phases: static and dynamical problems. Preprint, 2004.

[29] J. PaVel, M. Glogarova, and S. S. BAWA, Dielectric permittivity of ferroelectric liquid crystals influenced by a biasing electric field, Ferroelectrics, 76 (1987), pp. 221-232.

[30] J. Pavel and M. Glogarova, The effect of biasing electric field on relaxations in FLC investigated by the dielectric and optical methods, Ferroelectrics, 121 (1991), pp. 45-53.

[31] J. PAVEL, Behaviour of thin planar Sm $C^{*}$ samples in an electric field, J. Physique, 45 (1984), pp. 137-141.

[32] S. A. PIKIN, Structural Transformations in Liquid Crystals, Gordon and Breach Science Publishers, New York, 1991.

[33] H. R. Brand, P. E. Cladis, and H. Pleiner, Fluid Biaxial Banana Smectics: Symmetry at Work, Liquid Crystal Today 9 (1999), pp. 1-10. (1998), pp. 347-353.

[34] H. R. Brand, P. E. Cladis, And H. Pleiner, Symmetry and defects in $C_{M}$ phase of Polymeric Liquid crystals, Macromolecules, 25 (1992), pp. 7223-7226.

[35] Y. Shu And K. Bhattacharya, Domain patterns and macroscopic behavior of ferroelectric materials, Phil. Mag. B, 81 (2001), pp. 2021-2051.

[36] R. A. Sones, R. Petschek, D. W. Cronin, and E. M. Terentjev, Phys. Rev. E, 53 (1996), pp. 3611-3617.

[37] I. Stewart, The Static and Dynamic Continuum Theory of Liquid Crystals, Taylor and Francis, 2004.

[38] J. V. Stewart, Intermediate Electromagnetic Theory, World Scientific, Singapore, New Jersey, London and Hong Kong, 2001.

[39] T. P. Rieker, N. A. Clark and S. Lagerwall, Chevron local layer structure in surface stabilized ferroelectric smectic-C cells, Phys. Rev. Lett., 59 (1987), pp. 2658-2672. 